\title{
Delayed Reconstruction of Post Traumatic Ankle Malunion: A case report
}

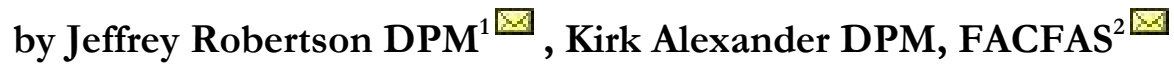

The Foot and Ankle Online Journal 4 (9): 3

Treatment of acute ankle fractures is clear in the approach; however, questions about delayed repair of chronic malunited ankle fractures still remain. Illustrated here is a case report of a 49 year old female that presented with a bimalleolar malunion, with severe lateral talar displacement and valgus position. She presented to our clinic 8 months following the initial injury. In the presence of mild to moderate degenerative changes at the tibio-talar articulation open reduction internal fixation (ORIF) was performed without complication. Proper anatomic alignment was established and stable fixation achieved.

Key words: Ankle fracture, malunion, tibio-talar articulation, delayed reconstruction.

Accepted: August, $2011 \quad$ Published: September, 2011

This is an Open Access article distributed under the terms of the Creative Commons Attribution License. It permits unrestricted use, distribution, and reproduction in any medium, provided the original work is properly cited. (The Foot and Ankle Online Journal (www.faoj.org), 2011 All rights reserved.

$\mathrm{I}$

$\mathrm{t}$ has been well documented that anatomic open reduction internal fixation (ORIF) of acute unstable ankle fractures decreases the rate and severity of post traumatic arthropathy compared to delayed intervention. ${ }^{1,3,5-15}$ Ankle fractures occur frequently with an unfortunate propensity for malunion if not treated, or under corrected. Despite the ankle joint's chondrocyte leniency toward increased demands, the ankle joint doesn't tolerate mal-alignment well. . $^{2,4}$

\section{Address correspondence to: The Swedish Podiatric Surgical Residency, Seattle, Washington 98122. \\ Email:jeff.robertson@swedish.org,kirka@pacmed.org}

\footnotetext{
${ }^{1}$ Jeffrey Robertson DPM, PGY-2. 747 Broadway, Swedish Podiatric Surgical Residency, Seattle, WA 98122. 206-320-5301.

${ }^{2}$ Kirk Alexander DPM, FACFAS. Surgical attending with Swedish Podiatric Surgical Residency, Seattle, WA 98122.
}

Early contact studies of ankle joint congruency show that a deviation of the talus by $1 \mathrm{~mm}$ may reduce tibiotalar contact surface area by as much as forty two percent. $^{10,11}$ These classic articles lower the threshold for intervention when we consider that a high percentage of malunions ultimately lead to debilitating post traumatic arthropathy.

Most commonly, Weber types B and C fractures result from external rotation and/or eversion forces shortening and externally rotating the fibula allowing the talus to shift laterally within the talocrural articulation. Untreated fractures or those with a medial malleolar fracture may allow for progressive rearfoot eversion and limited ankle joint dorsiflexion leading to further deformity and articular damage. 


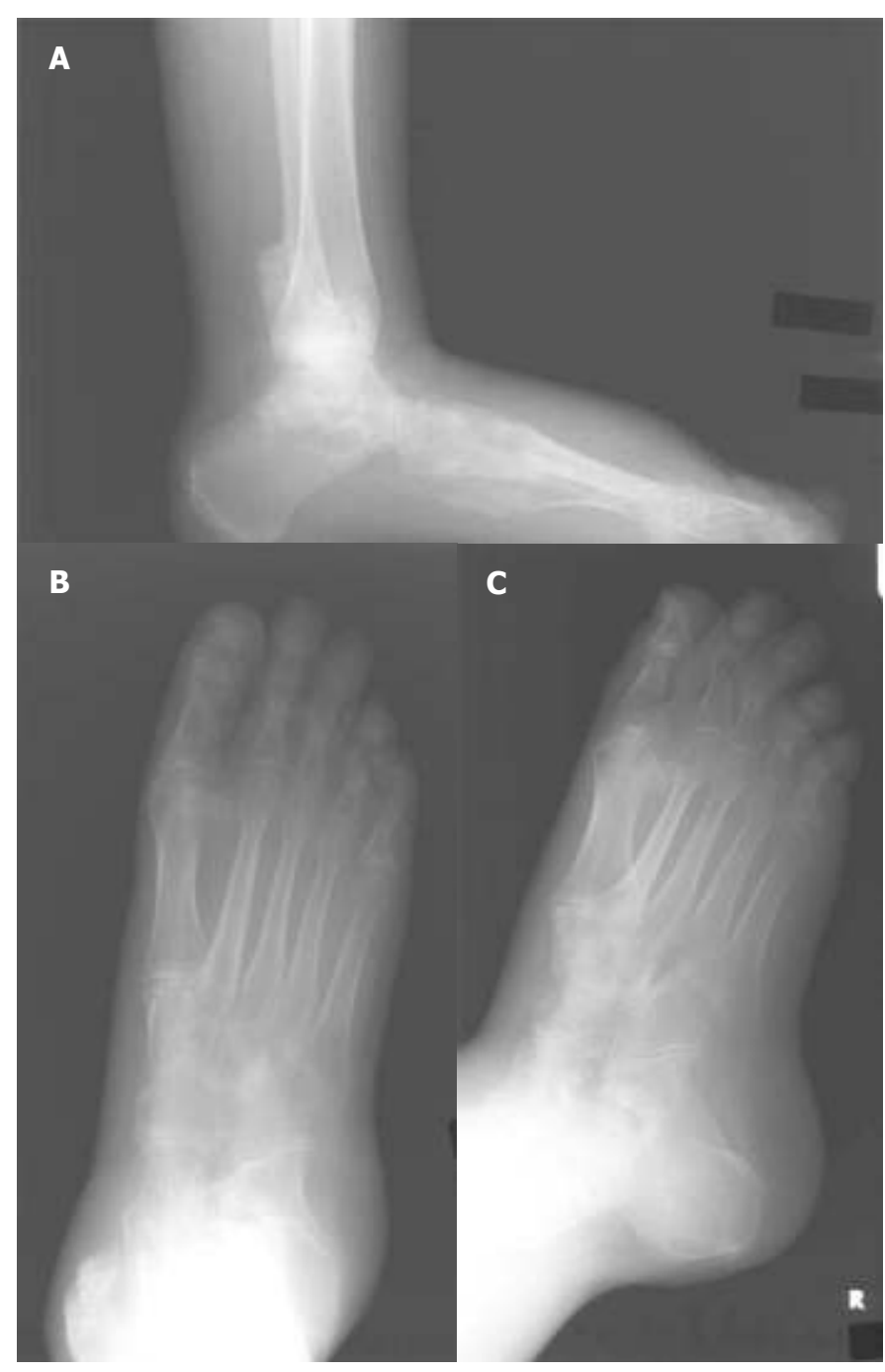

Figure 1 Initial Weight bearing plain foot radiographs. Lateral (A), dorsoplantar (B) and medial oblique (C) views of the foot at initial presentation.

Therefore, should all fractures be fixated? Should consideration be given to the time from injury to surgical intervention? What are the outcomes? Are there factors that should be considered during evaluation to improve surgical decision making and prognosis of such injuries? Presented here is a case study of an untreated bi-malleolar malunion approximately 8 months after initial injury.

\section{Case Report}

We discuss a 49 year old female who sustained a right ankle fracture in July of 2010. Prior to injury she was a community ambulator. She was initially treated at an outside facility with immobilization and limited weight-bearing. After healing of the fractures, she began limited ambulation. Due to severe valgus position of the ankle and rearfoot, she walked on the medial foot. This was awkward and uncomfortable, so walking and standing was limited. Therefore, she often used a wheelchair as a substitute for ambulation. Past medical history includes a symptomatic cerebral aneurysm which occurred in 1986.

During the physical examination her neurological presentation illustrated a symmetrical reduction in strength $(+4 / 5)$ to the anterior, lateral, and posterior compartments as well as to the intrinsic muscles of the legs and feet. However, no focal deficits were identified during the examination and protective sensation was intact bilateral and symmetrical.

Her vascular examination showed bilateral lower extremity pulses were palpable and symmetrical with +2 pitting edema. Doppler exam showed biphasic or triphasic pulses of the dorsalis pedis, perforating peroneal and posterior tibial arteries bilaterally. Dermatological exam demonstrates a superficial abrasion, 1 centimeter in diameter, over the medial malleolar malunion. Her contralateral leg had recently been injured in a fall secondary to the imbalance created from her malunion. The resulting wound over the left mid anterior tibia was large, with a mixed wound bed predominantly granular in nature. Neither wound showed signs of infection.

The musculoskeletal examination of the right lower extremity showed relative pain free passive ankle range of motion, with normal plantarflexion, but limited dorsiflexion. However, active range of motion showed increased sagittal plane motion illustrating greater midfoot and forefoot compensation. Mild to moderate crepitus of the ankle joint was appreciated. 


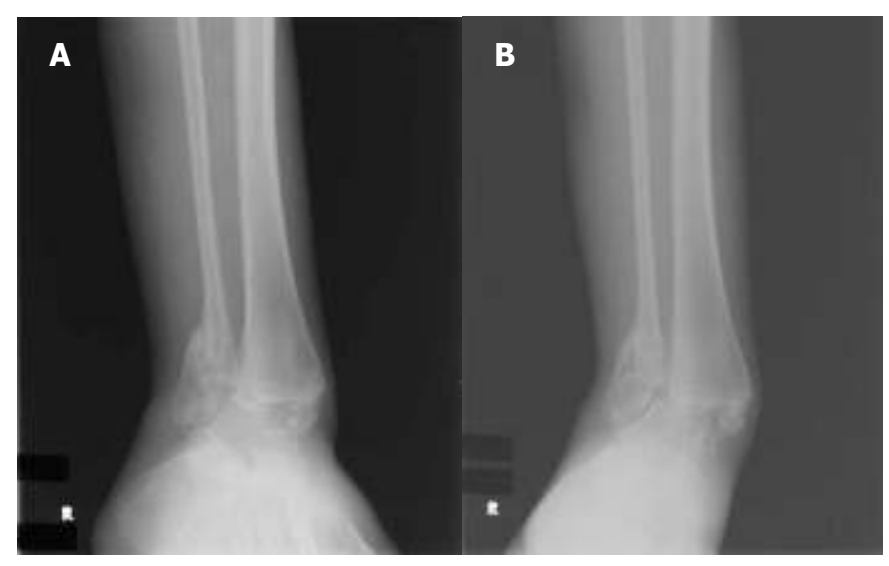

Figure 2 Initial anteroposterior (A) and oblique (B) weightbearing plain ankle radiographs.

The right foot in a maximal dorsiflexed position was 10 degrees plantarflexed with the knee extended and flexed. The subtalar joint, midtarsal joint, first ray and metatarsal phalangeal joints were flexible, and without crepitus or impingement. Radiographs demonstrated 30-35 degrees of ankle valgus with the talocalcaneal position maintained within anatomic alignment. Therefore, during ambulation axial load was translated medially in relation to the rearfoot. This deformity was fully compensated by the flexible degree of midfoot and forefoot supinatus and abduction.

Initial plain radiographs illustrate malunion of a right Weber B bi-malleolar ankle fracture. Medial and lateral malleolar fragments were translated and angulated laterally. No gross deformities were noted of the distal forefoot, midfoot, or rearfoot. (Figs. 1A$1 \mathrm{C}$ and $2 \mathrm{~A}$ to $2 \mathrm{~B}$ ) To further investigate the degree of deformity a CT scan was performed. (Figs. 3A and 3B) The coronal and sagittal images illustrate the incomplete malunion at both malleoli as well as the presence of exostoses/calcification at the anterolateral aspect of the ankle joint and syndesmosis. (Fig. 4A and 4B) Tibio-talar joint space remained adequate. There was no significant subchondral sclerosis, or cystic changes.

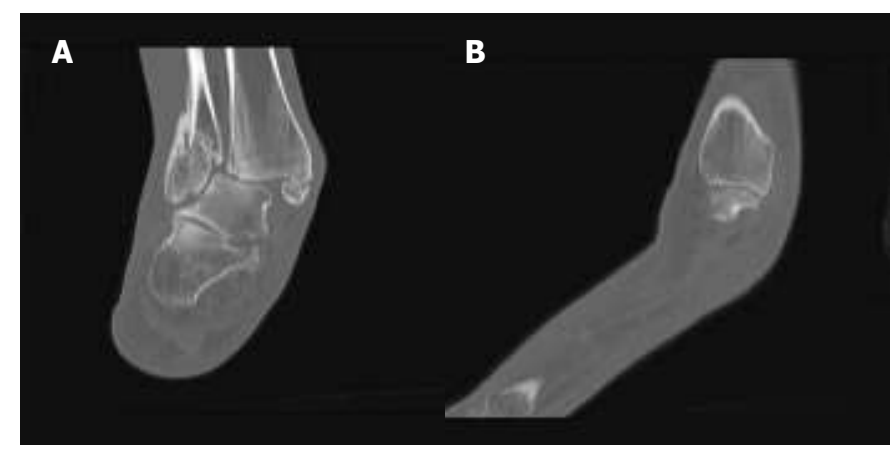

Figure 3 Computed tomography radiographs illustrating the coronal image illustrating bi-malleolar ankle fracture with severe valgus deformity at the ankle joint. (A) The sagittal image of the medial malleolus showing incomplete union of its distal aspect. (B)

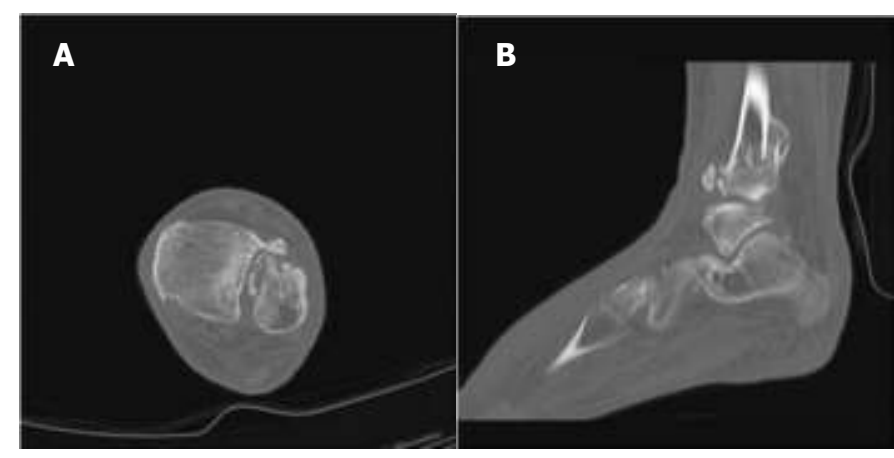

Figure 4 Computed tomography adiographs illustrating the axial (A) and sagittal (B) images showing exostosis vs possible Wagstaff avulsion fracture with ossification of the distal tibial fibular syndesmosis.

The position of the ankle did not allow normal function or stability therefore, we felt surgical intervention was appropriate considering severe ankle translation and angulation, pre-ulcerative lesion secondary to prominent medial malleolus, and minimal evidence of articular degeneration. Alternatives were discussed including but not limited to arthrodesis and bracing, but patient elected to forward with ORIF. Consent was obtained prior to procedure. 


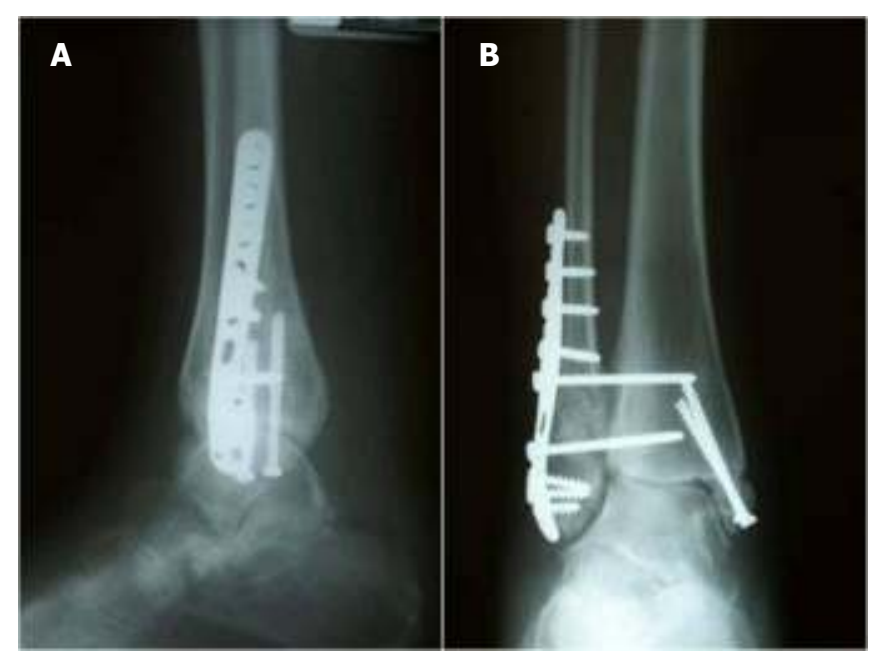

Figure 5A and 5B General radiographs four weeks after surgery. Lateral $(A)$ and $A P(B)$ views are shown.

\section{Procedure}

Preoperative antibiotics were administered (2g cefazolin) and general anesthesia with a popliteal block to the right lower extremity was performed. Saphenous coverage was provided by a separate local injection. A bump was placed under the ipsilateral hip. A right thigh tourniquet was applied. The right lower extremity was prepped and draped in the usual sterile fashion. Prior to incision the extremity was exsanguinated and the tourniquet was inflated to $275 \mathrm{mmHg}$.

A percutaneous incision was made along the Achilles tendon where a percutaneous lengthening procedure was performed. At the distal third of the fibula a longitudinal incision was made slightly anterior to its midline as to provide adequate visualization of the fibula, the anterolateral ankle joint and syndesmosis.

The reactive bone formation was removed from the distal fibula and contoured with a ronguer and hand rasp. Removal of the calcifications from the anterior syndesmosis was also performed allowing for better tibio-fibular reduction.
The distal fibular fragment was liberated from its fibro-osseous union by re-creating the fracture-line. A $1.5 \mathrm{~mm}$ drill bit was utilized to perforate the length of the fracture followed by the use of a small straight osteotome to connect the drill holes, recreating the original fracture orientation. The fibrous tissue was removed from within the fracture site as well as off the most lateral aspect of the fibula. The lateral aspect of the tibiotalar articulation could now be inspected. Of note, was the lack of significant articular degeneration or presence of osteochondral lesions to the lateral talar shoulder. However, there were mild erosive changes to the lateral tibial plafond. Prior to fibular reduction and fixation, the medial malleolus was addressed to facilitate the correction of ankle translation and angulation.

A curvilinear incision was created anterior to the midline of the medial malleolus. This approach was performed to avoid placing the incision through the superficial abrasion at the medial malleolus. A transverse periosteal incision was created directly over the fracture line. A power saw was used to transect the malunion. A significant amount of fibrous tissue was found within the medial gutter and tightly adherent to the medial malleolar articular cartilage. All the fibrous tissue was removed by combination of sharp dissection and ronguer. Underlying the fibrous cap at the medial malleolus was healthy articular cartilage. Also noted was the isolated presence of mild to moderate erosive changes to the central talar dome as it was the focal point of weight bearing against the lateral tibial plafond.

After mobilizing the lateral and medial malleoli, the rearfoot was medialized. Intra-operative fluoroscopy guided medial malleolar reduction and fixation. Lag technique was utilized to provide interfragmentary compression that stabilized the medial malleolus and prevented lateralization secondary to soft-tissue contracture. The lateral malleolus was reduced and stabilized with temporary fixation. A large void at the fibular fracture was filled with morselized cancellous allograft. Fibular fixation was completed with a Zimmer's Periarticular Distal Lateral Fibular Plate. 
The fracture void and bone graft prevented placement of standard fibular interfragmentary screws, therefore, two syndesmosic [syndesmotic] screws were placed through the plate for increased stability to the fragments and rigidity of the construct. Final inspection and irrigations were performed followed by layered closure. The patient was placed in a well padded modified posterior Jones compression splint. The patient tolerated the procedure well and without complication. The patient was placed on a strict non-weight bearing status and discharged to a skilled nursing facility to aid in her post operative recovery period.

Stability achieved by the internal fixation and preservation of anatomic alignment in light of severe angular deformity four weeks post operatively. (Fig. $5 \mathrm{~A}$ and $5 \mathrm{~B}$ ) Both the lateral and medial malleoli have maintained their positions well. Trabeculation with consolidation is appreciated at the medial and lateral malleoli. The fibula is near anatomic. The patient has progressed.

\section{Discussion}

Ankle fractures are common, and foot and ankle surgeons have developed and refined skills to successfully aid their patients in recovering their activities of daily living). Conceptually, we consider the ankle as a stable construct when all the ligaments and bony architecture remain intact creating the "stable ring"." When a single break in the ring occurs, such as in a Weber A, B or C fracture, the ankle construct is still considered to be stable and surgical intervention may not be necessary. However, when there is additional damage such as additional ligamentous or osseous injury, resulting in a second "break" within the ring, the ankle joint is considered to be unstable warranting surgical stabilization for optimal prognosis. For best possible functional results it is widely accepted to reduce the articulations anatomically through direct surgical visualization and fixation. ${ }^{6}$
Complications come to mind when thinking about malunions of the ankle joint, such as degree of deformity, presence of degenerative arthropathy, patient level of activity, age, patient specific goals and overall surgical candidacy. Despite the obvious hesitation to perform delayed reconstruction early studies have illustrated that a patient's age makes no difference and that the importance of maximizing talocrural articulation even in the presence of mild arthritic changes is suggested. ${ }^{7,14}$ In fact, arthritic changes should not be considered a contraindication, and any presence of articular degeneration should be the very reason for intervention. ${ }^{7,11}$ Reidsma's and colleagues $^{11}$ illustrate better long term prognosis for patients when there was less time between initial injury and reconstruction. Marti and his colleagues included one caveat to the above, that degree of deformity and age did not alter outcome as long as the patient's functional status and meticulous preoperative planning was performed. This allows full understanding of the nature of the deformity and requirements to recover fibular length, rotation and achieve anatomic tibio-talar congruency.

Many studies illustrate successful correction of ankle malunions. The transverse fibular osteotomy has been widely established to regain fibular length. ${ }^{1,6,10,11-}$ 15 This is common in Weber $\mathrm{C}$ malunions. However, re-creation of the original fracture has only been mentioned in malunions with length and rotational components. This may be more attributable to the necessity of meticulous pre-operative planning so as to re-create the correct fracture pattern to allow proper lengthening and de-rotation of the distal fragment simultaneously. In this particular case of a Weber B malunion, correction of length and rotation was facilitated by recreating the original fractures. In addition to liberating each fragment, fixating the medial malleolar fragment first facilitated medialization of the talus within the mortise allowing for correct fibular fixation and overall anatomic alignment 
This case study continues to support open correction of malunited ankle fractures. We agree with current literature that delayed repair is preferred, giving less regard to degree of deformity and articular degeneration.

We feel arthrodesis and arthroplasty should be reserved as salvage procedures for progressive deformity or failed delayed repair. The approach described in this case study may benefit delayed reconstruction of malunited ankle fractures by open liberation of the malleolar fracture fragments, recreation of a Weber B fracture, followed by fixation of the medial malleolus, and then lateral malleolus. This approach may allow for better medialization of the talus and allowing anatomic alignment of talocrural joint.

\section{References}

1. Davis JL, Giacopelli J. Transfibular osteotomy in the correction of ankle joint incongruity. J Foot Ankle Surg 199534 (4): 389-399.

2. Fetter NL, Leddy HA, Guilak F, Nunley JA. Composition and transport properties of human ankle and knee cartilage. J Orthop Res 2006 24: 211-219.

3. Henderson WB, Lau JT. Reconstruction of failed ankle fractures. Foot Ankle Clin 2006 11: 51-60.

4. Hendren L, Beeson P. A review of the differences between normal and osteoarthritis articular cartilage in human knee and ankle joints. Foot (Edinb) 2009 Sep;19(3):171-6.

5. Loder BG, Frascone ST, Wetheimer SJ. Tibiofibular arthrodesis for malunions of the talocrural joint. J Foot Ankle Surg 1995 34: 283-288.

6. Mann RA, Coughlin MJ, Saltzman CL. Surgery of the Foot and Ankle. $8^{\text {th }}$ edition; Volume II, Mosby Elsevier 2007.

7. Marti RK, Raaymakers EL, Nolte PA. Malunited ankle fractures. The late results of reconstruction. JBJS 1990 72B: 709-713.

8. Miller SD. Late reconstruction after failed treatment for ankle fractures. Orthop Clin North Am 1995 26: 3363-3373.

9. Neer CS. Injuries of the ankle joint: Evaluation. Conn State Med J 1953 17: 580-583.

10. Perera A, Myerson M. Surgical techniques for the reconstruction of malunited ankle fractures. Foot Ankle Clin 2008 13:737-751.

11. Ramsey PL, Hamilton W. Changes in tibiotalar area of contact caused by lateral talar shift. JBJS 1976 58A: 356-357.
12. Reidsma II, Nolte PA, Marti RK, Raaymakers ELFB. Treatment of malunited fractures of the ankle. A long term follow up of reconstructive surgery. JBJS 2010 92B: 66-70. 13. Sinha A, Sirikonda S, Giotakis N, Walker C. Fibular lengthening for malunited ankle fractures. Foot Ankle Int 2008 29: 1136-1140.

14. Ward AJ, Ackroyd CE, Baker AS. Late lengthening of the fibula for malaligned ankle fractures. JBJS 1990 72B: 714-717. 15. Weber BG, Simpson LA. Corrective lengthening osteotomy of the fibula. Clin Orthop Relat Res 1985 199: 61-67.

16. Weber D, Freiderich NF, Muller W. Lengthening osteotomy of the fibula for post-traumatic malunion. Indications, technique and results. Int Orthop 1998 22: 149-152. 